\title{
Modelo para análise ex ante de políticas de logística urbana baseadas em centros de distribuição urbanos: uma abordagem utilizando dinâmica de sistemas
}

Model for ex-ante analysis of city logistics policies based on urban distribution centers: an approach using system dynamics

Reinaldo Daniel Fioravantila,b] [1] , Orlando Fontes Lima Junior [a]

[a] Universidade Estadual de Campinas (UNICAMP), Faculdade de Engenharia Civil, Arquitetura e Urbanismo, Campinas, SP, Brasil

[b] Banco Interamericano de Desenvolvimento, Departamento de Infraestrutura e Energia, Washington, DC, EUA

Como citar: Fioravanti, R. D., \& Lima Junior, O. F. (2019). Modelo para análise ex ante de políticas de logística urbana baseadas em centros de distribuição urbanos: uma abordagem utilizando dinâmica de sistemas. urbe. Revista Brasileira de Gestão Urbana, 11, e20170170. https://doi.org/10.1590/2175-3369.011.002.A003

\section{Resumo}

O aumento do número de veículos de carga nos centros urbanos causa diversas externalidades negativas sob os aspectos financeiro, ambiental e econômico, por isso a formulação de políticas públicas que buscam otimizar a logística urbana vem ganhando importância nas agendas dos gestores públicos. Nesse contexto, este artigo tem o objetivo de propor um modelo para apoiar a formulação de políticas públicas de logística urbana com ênfase nos Centros de Distribuição Urbanos (CDUs). 0 método usado é a simulação matemática contínua baseado em dinâmica de sistemas e que incorpora elementos de gestão de estoque e operações. Este artigo apresenta o modelo e o procedimento proposto para sua aplicação em duas cidades hipotéticas, comparando a implantação de CDUs com outras políticas públicas. Os resultados, medidos pelos custos logísticos totais e pelas emissões de gases de efeito estufa (GEE), indicam que as restrições à circulação de veículos de carga tendem a impactar negativamente ambos os indicadores; por outro lado, os CDUs são soluções robustas que podem reduzir tanto os custos como as emissões e superam outras políticas, mas se aplicam a cidades que atingiram níveis de congestionamento críticos. A replicação do modelo e do procedimento é discutida.

Palavras-chave: Logística urbana. Centros de Distribuição Urbanos. Dinâmica de sistemas. Políticas públicas. Logística da cidade.

\begin{abstract}
The increase in the number of freight vehicles in urban areas generates several negative externalities from the financial, environmental and economic perspectives, and the formulation of public policies that optimize urban logistics has gained importance in the agenda of public managers. Thus, this paper purposes a model to support the formulation of public policies focused on urban logistics, with emphasis on Urban Distribution Centers (UDCs). The method used is mathematical simulation based on System Dynamics and incorporates elements of inventory management and operations management. This paper presents the model and the proposed procedure for its application in two hypothetical cities, and compares
\end{abstract}

RDF é doutorando, e-mail: reinaldof@iadb.org

OFLJ é professor titular, e-mail: oflimaj@fec.unicamp.br 
the adoption of UDCs with other policies. The results, measured by total logistics costs and greenhouse gases (GHG) emissions indicate that the restrictions tend to negatively impact both indicators. On the other hand, UDCs are robust solutions that can reduce both costs and emissions and outperform other policies in the case of cities that have reached critical congestion levels. The replicability of the model is discussed.

Keywords: Urban logistics. Urban Distribution Centers. System dynamics. Public policies. City logistics.

\section{Introdução e motivação}

O aumento de veículos de carga nos centros urbanos causa diversas externalidades negativas, como o aumento do congestionamento, das emissões de gases de efeito estufa (GEE) (Thompson, 2015) e da demanda por espaços para carga e descarga (Holguín-Veras et al., 2008; Rodrigue et al., 2013), bem como a deterioração mais rápida da infraestrutura de transporte (Crainic et al., 2009). Estima-se que os níveis de emissões de GEE gerados pelo transporte de cargas nas grandes cidades podem variar entre 15 e $30 \%$ de todas as emissões urbanas provenientes do setor de transportes (Dablanc, 2007). Por outro lado, os custos da chamada última milha representam $28 \%$ dos custos totais da cadeia de distribuição das empresas (Goodman, 2005).

Historicamente, as políticas públicas de mobilidade urbana não incluíam o transporte de carga, o qual era uma preocupação delegada ao setor privado (Wolpert \& Reuter, 2012). No entanto, por causa do elevado nível de urbanização, do crescimento do comércio eletrônico e da tendência das empresas de buscar um maior nível de serviço a seus clientes, implicando entregas mais frequentes, tem se tornado essencial a implementação de políticas públicas que busquem otimizar o transporte de cargas no ambiente urbano, também denominado "logística urbana".

Várias políticas públicas têm sido propostas para melhorar a eficiência e reduzir as externalidades da logística urbana, como as restrições à circulação de caminhões (Holguín-Veras et al., 2014), o aumento do fator de ocupação de carga, a introdução de limites para o tamanho e o peso de veículos, a implantação de Centros de Distribuição Urbanos (CDUs) (Allen et al., 2012; Quak \& Tavasszy, 2011; Marcucci \& Danielis, 2008), entre outros. 0 foco principal deste artigo são os CDUS, que são infraestruturas logísticas localizadas em áreas urbanas, ou nas proximidades delas, nas quais as empresas realizam o transbordo e a consolidação dos produtos que serão posteriormente distribuídos nos centros urbanos (Allen et al., 2014).

Dada a variedade de opções de políticas públicas e a presença de múltiplos agentes (varejistas, distribuidores, fabricantes e população), a tomada de decisão por parte dos gestores públicos se torna complexa, e os custos por uma decisão errada são altos (Anand et al., 2012). Nesse contexto, o objetivo do presente trabalho é contribuir para a tomada de decisão em logística urbana de duas maneiras: i) oferecer insights sobre os benefícios e a robustez dos CDUs comparativamente com outras políticas; ii) propor um modelo e um procedimento para sua implementação para apoiar os gestores públicos na avaliação prévia (ex ante) dos resultados esperados da implantação de CDUs.

As hipóteses testadas são que os benefícios gerados pelos CDUs podem levar longo tempo de maturação e dependem de características de cada cidade, como o nível de congestionamento e a intensidade do transporte urbano de mercadorias, o que pode explicar a dificuldade em mantê-los sustentáveis (Browne et al., 2005; Van Duin et al., 2012; Holguín-Veras et al., 2014). Além disso, como contribuição à literatura, o modelo proposto incorpora políticas de gestão de estoque para representar o processo de decisão individual das empresas, as quais, ao depararem-se com o aumento dos custos de transporte, podem realizar reorganizações na sua distribuição (FHA, 2008) tanto no curto prazo, com a mudança dos lotes de entrega e da frota, como no longo prazo, com o aumento ou a redução dos centros de distribuição.

As próximas seções do artigo incluem uma revisão da literatura relacionada às políticas públicas de logística urbana, aos CDUs e aos modelos de apoio à decisão em logística urbana, seguida pela apresentação do modelo proposto e dos resultados da simulação, além do procedimento para sua aplicação. A última seção apresenta reflexões sobre as implicações na adoção de políticas públicas. 


\section{Políticas de logística urbana}

As políticas públicas em logística urbana têm sido documentadas na literatura desde os anos de 1980, principalmente na Europa e no Japão. Button \& Pearman (1981) destacaram como fatores motivadores o crescimento no tráfego de caminhões, resultado do crescimento econômico das cidades, as limitações para expansão da infraestrutura e o aumento das preocupações ambientais. Ogden (1992) propôs uma classificação das políticas de logística urbana em seis categorias de acordo com objetivos buscados: i) de eficiência; ii) econômicas; iii) de segurança viária; iv) ambientais; v) relacionadas a infraestruturas (por exemplo, reduzindo custos de manutenção das vias); vi) relacionadas a infraestruturas urbanas (por exemplo, preservando centros históricos). Visser et al. (1999) discutiram a evolução das políticas aplicadas na Europa de 1980 a 1999 e destacaram duas abordagens: a micro, quando é liderada pelo setor privado, e a macro, quando é guiada pelo setor público. Eles concluíram que o melhor resultado se obtém com uma combinação das duas. Lindholm (2013) destacou que, embora fosse, no início, uma preocupação das grandes cidades, a inclusão da carga no planejamento de transportes de cidades médias e pequenas começa a ganhar importância.

As políticas e as intervenções mais comuns apresentadas na literatura incluem as restrições à circulação de caminhões (Holguín-Veras et al., 2014), a melhora da roteirização dos veículos (HolguínVeras et al., 2015), o aumento do fator de ocupação de carga, a introdução de limites para o tamanho e o peso de veículos (Qureshi et al., 2013), a implantação de pick-up points (Visser et al., 2014; Weltevreden, 2008), a implantação de zonas de baixas emissões (Browne et al., 2005; Giuliano \& Dablanc, 2013) e a implementação de Centros de Distribuição Urbanos (CDUs) (Allen et al., 2012; Quak \& Tavasszy, 2011; Marcucci \& Danielis, 2008).

\section{Centros de Distribuição Urbanos (CDUs)}

A implantação de CDUs apresenta grande potencial para atingir os objetivos da logística urbana (Van Duin et al., 2012), como a redução do número de veículos/km percorrido (Panero \& Shin, 2011), o aumento da ocupação de carga, a melhor adaptação às janelas de tempo (Taniguchi et al., 2012) e a colaboração entre empresas (Janssen \& Oldenburger 1991; Van Duin, 1997; Taniguchi et al., 1999).

O conceito dos CDUs tem suas origens nos anos de 1970 com estudos de viabilidade no Reino Unido, no Japão, nos Estados Unidos e no Canadá, com os primeiros projetos tendo sido implantados na França e na Holanda (Allen et al., 2012). Nos anos de 1990, houve novamente um crescente interesse pelos CDUs, especialmente no Japão, sob a denominação Terminais Logísticos Urbanos Públicos (TLUs), na Holanda (Janssen \& Oldenburger, 1991) e na Alemanha (Ruske, 1994).

0 potencial dos CDUs é quase consenso na literatura, com estudos de viabilidade ex ante positivos (Marcucci \& Danielis, 2008) e sólidas evidências sobre os benefícios em termos de redução de veículos/km, congestionamento e emissões, podendo variar de 18 a 60\% (Kohler, 2004; Panero \& Shin, 2011; Van Duin et al., 2012), especialmente se implementados de maneira complementar a outras políticas, como pedágios urbanos e janelas de tempo (Dablanc, 2007). No entanto, os resultados de sua implantação no longo prazo não são consistentes, e a viabilidade econômica e financeira tem sido difícil de atingir.

Van Duin et al. (2010) avaliaram experiências na Holanda, na Alemanha, no Reino Unido, na Espanha e na França no período de 1994 a 2010 e concluíram que os CDUs que atingiram os benefícios de redução de viagens e emissões, na maioria dos casos, não chegaram aos resultados financeiros esperados. Entre os desafios para atingir a viabilidade financeira, incluem-se a alocação dos custos e dos benefícios entre diferentes agentes (Van Duin et al., 2010; Holguín-Veras et al., 2014) e a dificuldade de operar sem subsídios públicos (Browne et al., 2005; Van Duin et al., 2012), reforçando a necessidade de pesquisas que indiquem os benefícios dos CDUs com uma visão de longo prazo e que envolvam o poder público e o setor privado. 


\section{Tomada de decisão em logística urbana}

A falta de análises prévias (Comi et al., 2012) leva a muitas políticas e intervenções em logística urbana seguirem um processo de "aprender fazendo" (Visser et al., 1999), o que pode gerar resultados contrários aos buscados, como no caso das restrições aos caminhões (Quak \& De Koster, 2006; Meyer Sanches et al., 2013). Além disso, a experiência mostra que os modelos tradicionais utilizados no planejamento de transportes, como o modelo quatro etapas, não são adequados para representar as particularidades da logística urbana (Taniguchi \& Thompson, 2002), a qual requer uma perspectiva sistêmica que inclua as dimensões financeira, econômica, de satisfação dos clientes e de sustentabilidade ambiental (Lima, 2004). Nesse contexto, os modelos de logística urbana abordam o problema, principalmente, sob a perspectiva da otimização de veículos e rotas (Taniguchi et al., 2012; Figliozzi, 2007), dos fluxos de commodities (Holguín-Veras \& Patil, 2008) ou por meio de modelos mistos (Comi et al., 2012).

Modelos baseados em commodities estimam a demanda e a oferta de produtos e as convertem em viagens de carga (Wisetjindawat et al., 2007; Holguín-Veras \& Patil, 2008). Modelos baseados em veículos e rotas estimam diretamente o número de viagens por meio de fatores de atração, classificação de utilização do solo e dados socioeconômicos (Oppenheim, 1993; Hunt \& Stefan, 2007; Muñuzuri et al., 2010). Outros tipos de modelos analisam o comportamento individual das empresas, como em Friesz \& Holguín-Veras (2005) e Holguín-Veras et al. (2006), que utilizam modelos econômicos para analisar as condições necessárias para o sucesso de políticas, como entregas noturnas.

Comi et al. (2012) destacaram limitações nos métodos baseados em viagens e commodities e sugeriram o desenvolvimento de modelos mistos que descrevessem melhor os vínculos entre as políticas adotadas e o comportamento dos agentes.

Vários autores propuseram modelos para determinar a localização e o tamanho dos CDUs, como Taniguchi et al. (1999) e Muñoz-Villamizar et al. (2014). Esses últimos autores, por meio de um modelo de otimização, concluíram que, ao abordarem a localização dos CDUs em conjunto com a definição das rotas, podia-se alcançar 30\% de redução nos custos logísticos. Crainic et al. (2004) e Muñuzuri et al. (2012) propuseram a introdução de dois níveis de distribuição, sendo os CDUs principais combinados com plataformas satélites. Van Duin et al. (2012) fizeram simulação de agentes para avaliar a aderência do setor privado aos CDUs e apontaram a necessidade de subsídios públicos para sua viabilidade. Janjevic et al. (2015) utilizaram análise de cenários para avaliar a implementação de CDUs na Bélgica e concluíram que a metodologia era robusta para abordar várias incertezas presente nas análises; no entanto, o modelo apresentado era estático e não considerava a evolução do sistema ao longo do tempo.

\section{Utilização de Dinâmica de Sistemas (SD)}

O modelo proposto é baseado em dinâmica de sistemas (SD, da sigla em inglês de System Dynamics). A literatura apresenta a utilização de SD na modelagem de problemas de transporte e logística como ferramenta potente para avaliar políticas e estratégias setoriais (Abbas \& Bell, 1994), como políticas de introdução de veículos elétricos e de manutenção de rodovias (Shepherd, 2014). Além disso, a SD também é amplamente utilizada em problemas de gestão da cadeia de suprimentos (Angerhofer \& Angelides, 2000). Taniguchi et al.(2014) apresentaram a dinâmica de sistemas como ferramenta promissora para modelar a logística urbana, porém ainda são poucas as referências na literatura (Thaller et al., 2016), destacando-se Oliveira et al. (2010), que estudaram a aderência do setor privado a pontos de entregas inteligentes, e Meyer Sanches et al. (2013), que modelaram a resposta das transportadoras às restrições de veículos em São Paulo. Thaller et al. (2016) apresentaram um modelo conceitual em SD para modelar a logística urbana com foco na demanda e nos serviços de transporte, mas não apresentaram aplicação. 


\section{Descrição do modelo}

O modelo de simulação proposto (Figura 1) é composto por seis componentes: i) a demanda de transporte de carga, que é exógena ao sistema e acompanha o crescimento econômico da cidade e da economia; ii) o sistema de transporte, que relaciona a densidade de tráfego nas vias com a velocidade média dos veículos; iii) as decisões do setor privado, que incluem as decisões de lote econômico, tamanho dos caminhões e utilização ou não dos CDUs; iv) as políticas públicas; v) os investimentos em infraestrutura pública; e vi) os indicadores de desempenho.

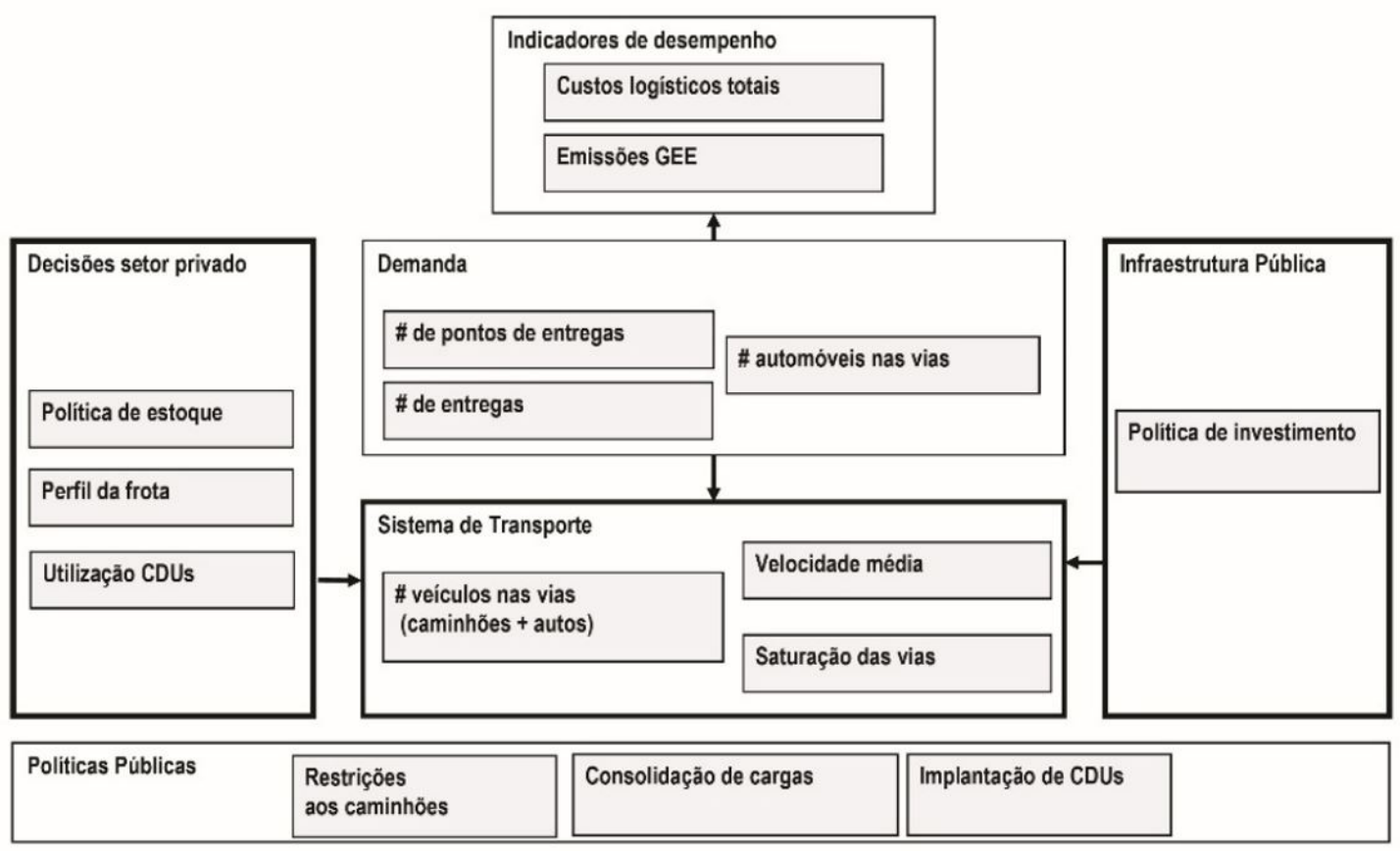

Figura 1 - Componentes do modelo. Fonte: elaborada pelos autores \# =número.

\section{Premissas e considerações sobre o modelo}

Como contribuição à literatura, o modelo internaliza algumas das variáveis que tipicamente são consideradas como exógenas: a decisão sobre o tipo de frota, apresentada como função do lote econômico; a decisão de abrir CDUs, como função dos custos de transporte e dos custos de estoque; e a velocidade média, como função da densidade de tráfego. As simulações incluem o fluxo de produtos (representado pelas entregas), o fluxo de veículos e o fluxo logístico resultante do comércio entre empresas, mas não as entregas do comércio eletrônico ou de outras atividades comerciais e industriais. Durante a aplicação do modelo, utilizaram-se cidades hipotéticas, com a calibragem e a configuração do cenário base apresentado na Tabela 1 de acordo com os dados coletados na cidade de Curitiba, Paraná, Brasil (IDB, 2013; IPPUC, 2014). 0 desenvolvimento do modelo de simulação utilizou o software Vensim, versão PLE x32.

Tabela 1 - Probabilidade de utilizar caminhões leves $\left(\lambda_{t}\right)$ como função do EOQ

\begin{tabular}{cccccc}
\hline EOQ (tons) & $\lambda_{t}$ & EOQ (tons) & $\lambda_{t}$ & EOQ (tons) & $\lambda_{t}$ \\
\hline 3,00 & 0,50 & 5,25 & 0,20 & 7,50 & 0,12 \\
3,75 & 0,34 & 6,00 & 0,16 & 8,25 & 0,10 \\
4,50 & 0,25 & 6,75 & 0,13 & 9,00 & 0,09 \\
\hline
\end{tabular}

Fonte: elaborada pelos autores. 


\section{Estrutura dinâmica do sistema}

A representação do modelo usando dinâmica de sistemas contém três principais ciclos causais: um ciclo que representa o sistema de transporte e dois ciclos que descrevem as decisões do setor privado. A seguir, detalha-se cada um desses ciclos (o modelo completo se apresenta no Anexo 1).

\section{Primeiro ciclo: sistema de transporte}

O primeiro ciclo (Figura 2) relaciona o congestionamento (medido pela densidade de tráfego) com a velocidade média e o número de caminhões nas vias: quando os níveis de congestionamento aumentam, a velocidade média nas vias diminui; logo, para cumprir com as entregas exigidas pelos clientes, mais caminhões são enviados às ruas, aumentando ainda mais o nível de congestionamento.

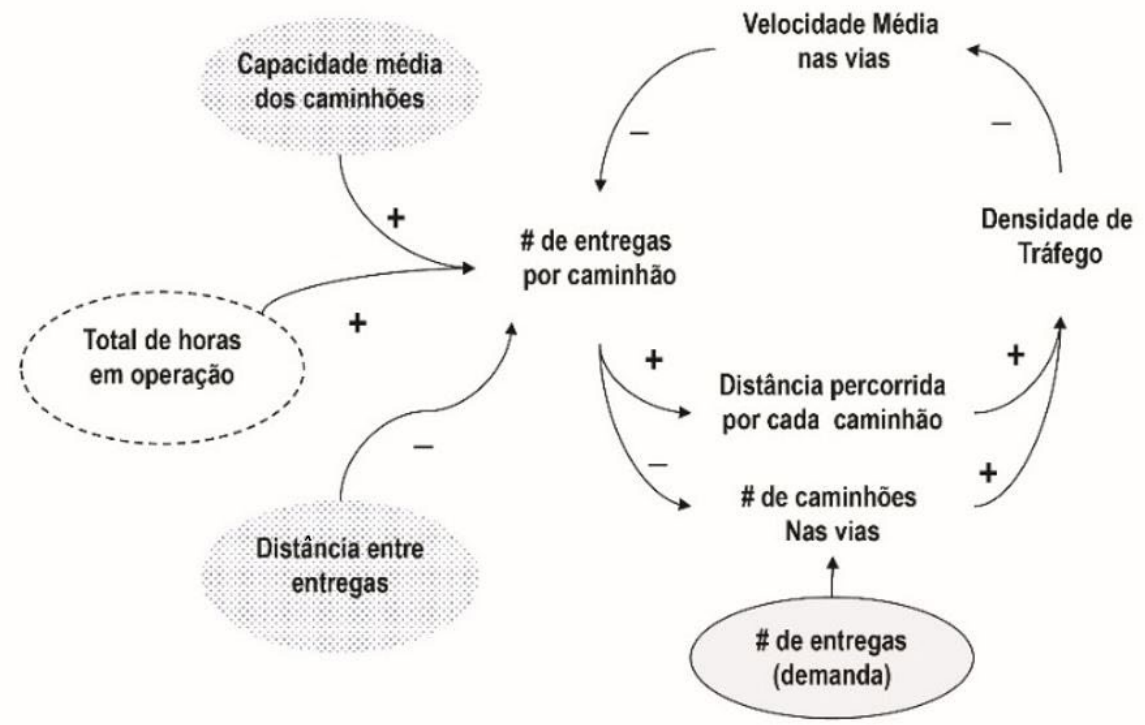

Variáveis exógenas
Decisōes setor privado
Politicas públicas

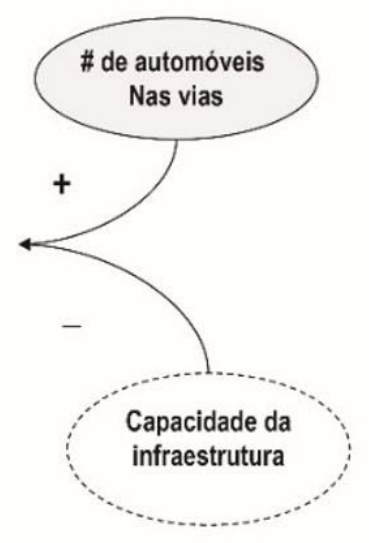

Calculada por outro componente do modelo

Figura 2 - Ciclo do sistema de transporte e distribuição \# =número. Fonte: elaborada pelos autores.

As Equações 1, 2 e 3 descrevem a relação entre as principais variáveis do primeiro ciclo:

A velocidade média $S$, medida em $\mathrm{km} / \mathrm{h}$, é função da densidade de tráfego (f) e da velocidade máxima nas vias $(60 \mathrm{~km} / \mathrm{h})$ por meio de uma função exponencial negativa (Karato \& Sato, 2009).

A densidade de tráfego $(f)$ é determinada pelo número de caminhões nas vias $\left(V_{t}\right)$, pela distância percorrida pelo caminhão $\left(L_{t}\right)$, pelo número de automóveis $\left(V_{a}\right)$, pela distância percorrida pelos automóveis $\left(L_{a}\right)$ e pela capacidade das vias $\left(C_{r}\right)$; o fator 0,5 é aplicado ao número de autos, assumindo que ocupam metade do espaço de um caminhão.

Finalmente, o número de entregas por caminhão por dia $\left(D / V_{t}\right)$ é dado pela velocidade média das vias $(S)$, pelo total de horas de operação $(H)$, pelo tempo médio por entrega $\left(T_{d}\right)$, pela distância em vazio $\left(L_{e}\right)$ e pela distância entre entregas $\left(L_{d}\right)$, limitado pela capacidade média dos caminhões $\left(C_{t}\right)$.

$S=60 \cdot e^{-2 f}$ 
$f=\operatorname{Min}\left(\frac{\left(V_{t}, L_{t}+V_{a} \cdot L_{a} \cdot 0,5\right)}{C r}, 1\right)$

$\frac{D}{V_{t}}=\operatorname{Min}\left(\frac{\left(S \cdot H \cdot\left(1-T_{d}\right)\right)-L_{e}}{L_{d}}, C_{t}\right)$

Os gráficos a seguir representam o comportamento da velocidade média em função da densidade de tráfego: a Figura 3 mostra os valores calculados pelo modelo, e a Figura 4, os apresentados na literatura por Geroliminis \& Daganzo (2007).

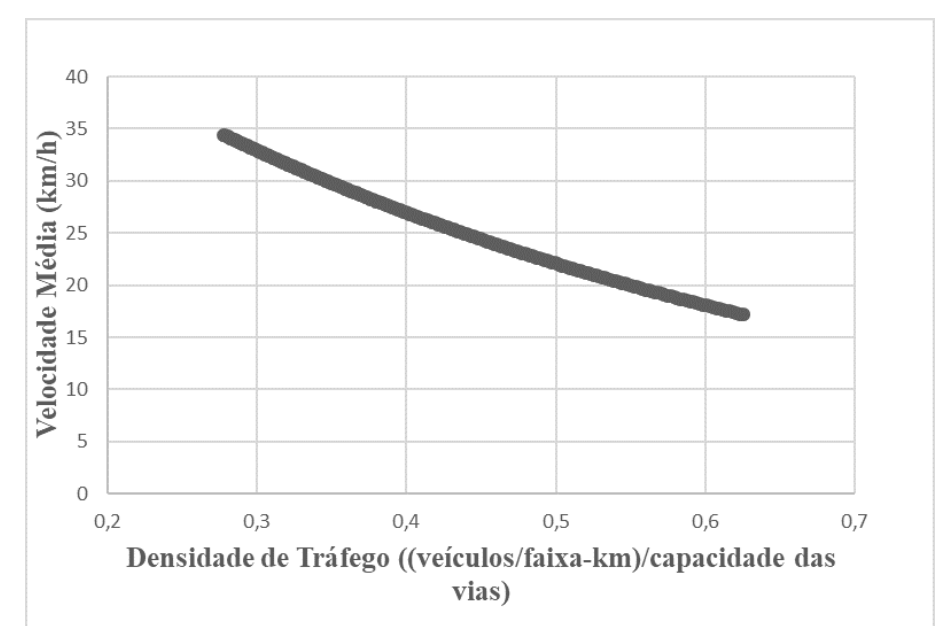

Figura 3 - Velocidade média (modelo). Fonte: resultados da simulação.

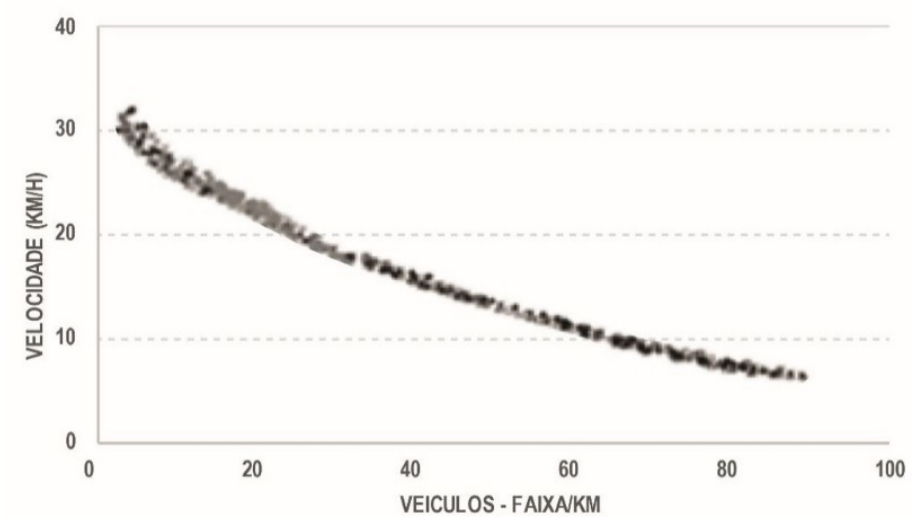

Figura 4 - Velocidade média (literatura). Fonte: Geroliminis \& Daganzo (2007).

Segundo ciclo: gestão de estoque e distribuição

O segundo ciclo (Figura 5) descreve a resposta do setor privado ao congestionamento: como a velocidade média diminui, o custo de transporte total aumenta em razão de mais consumo de combustível e mais tempo necessário para entregas; logo, de acordo com o modelo do lote econômico (EOQ, da sigla em inglês Economic Order Quantity), a reação racional das empresas seria aumentar o tamanho do lote mínimo de cada entrega (Combes, 2014), o que, por sua vez, aumentaria a utilização de veículos pesados 
(ou equivalentemente diminuiria a percentagem de veículos leves) e aumentaria a correspondente capacidade média dos caminhões.
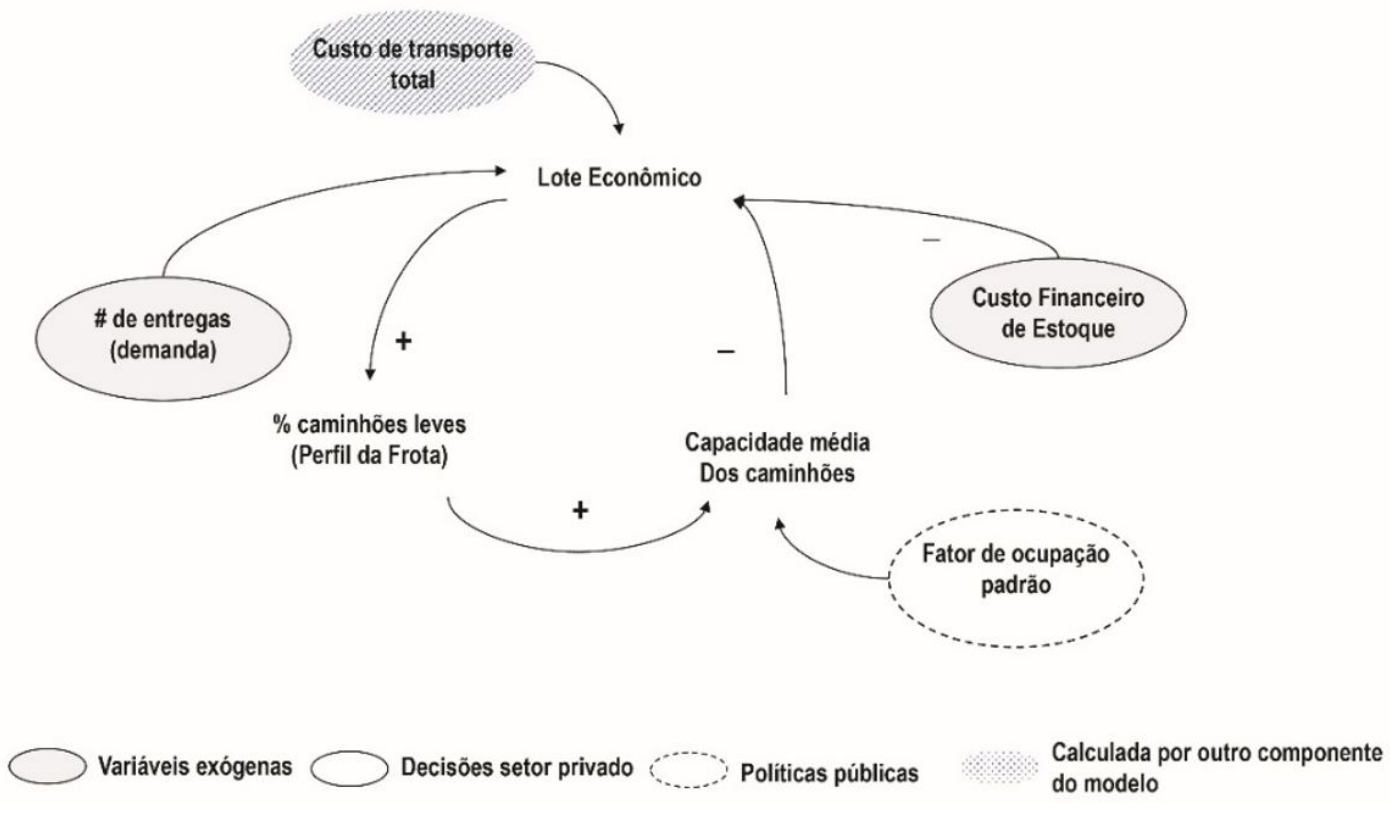

Figura 5 - Ciclo de gestão de estoque e distribuição \# =número. Fonte: elaborada pelos autores.

A Equação 4 descreve o cálculo do $E O Q$ para cada caminhão, dado pelo custo de transporte por entrega $(T / D)$, pela capacidade dos caminhões $\left(C_{t}\right)$, pelo número de entregas por ponto de venda $(D / P)$ durante o ano (250 dias) e pelo custo anual de estoque $(h)$.

Considerando que o EOQ não é o único fator que define o tamanho da frota, para estimar o percentual de caminhões leves $\left(\lambda_{t}\right)$ foi assumido uma curva normal, com média igual ao EOQ e desvio-padrão de $50 \%$, em que $\lambda_{t}$ é a probabilidade de utilizar um caminhão de 3 toneladas. A Tabela 1 descreve os valores do EOQ em número de entregas, em toneladas equivalentes, e o valor de $\lambda_{t}$ correspondente.

Finalmente, a Equação 5 descreve a capacidade média dos caminhões como função do percentual de caminhões leves $\left(\lambda_{t}\right)$ e fator de ocupação $(\varphi)$. Para os caminhões pesados, utilizou-se o valor de 6 toneladas, e o peso de cada entrega assumido foi de 0,15 toneladas.

$E O Q=\sqrt{\frac{\left(2 \cdot \frac{T}{D} \cdot C_{t}\right) \cdot\left(\frac{D}{P} \cdot 250\right)}{h}}$

$C_{t}=\frac{\left(3 \cdot \lambda_{t}+6 \cdot\left(1-\lambda_{t}\right)\right) \cdot \varphi}{0,15}$

\section{Premissas e considerações sobre o lote econômico}

Embora se reconheça como limitada a política de lote econômico no ambiente urbano, no qual prevalece a estratégia just in time ditada pelos varejistas, em muitos casos os fabricantes e os distribuidores continuam adotando o EOQ para seus processos produtivos de aquisição (Fazel, 1997). É possível notar na Figura 6 que, embora, com o aumento dos custos, o lote econômico ideal aumente e, portanto, a proporção ideal de caminhões leves diminua (frota ideal), não há mudança relevante no 
tamanho da frota real. Isso acontece porque, ao utilizar caminhões maiores (até 6 toneladas), não haveria possibilidade de fazer as entregas a tempo, então as empresas optam pelos veículos menores.

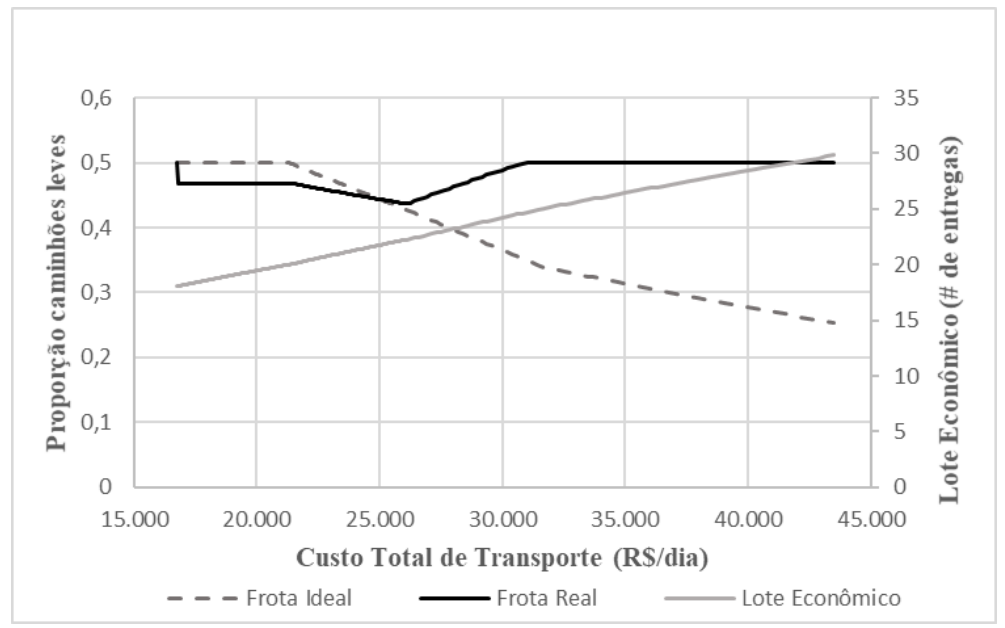

Figura 6 - Lote econômico e perfil da frota/custo de transporte. Fonte: resultados da simulação.

\section{Terceiro ciclo: os CDUs}

O terceiro ciclo (Figura 7) descreve a implantação dos CDUs por meio de uma equação de equilíbrio entre custos de transporte e custos de estoque, com a premissa de que esse equilíbrio converge para o ponto de custo logístico total ótimo (Chopra \& Meindl, 2004).

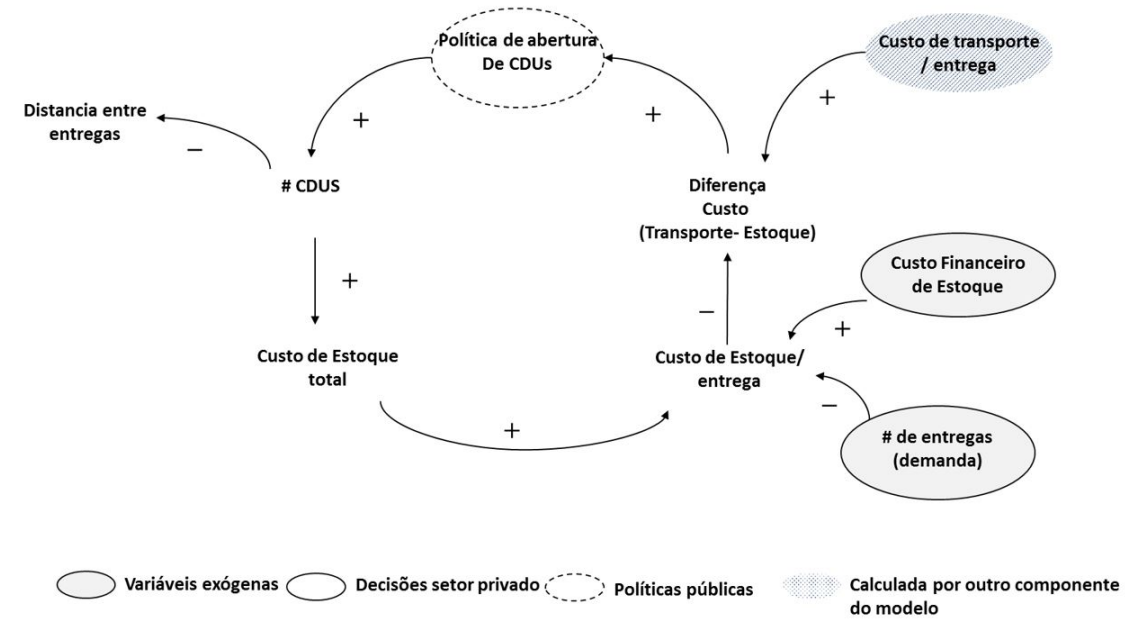

Figura 7 - Ciclo de implantação e de utilização dos CDUs \# = número. Fonte: elaborada pelos autores.

Com o aumento dos custos de transporte por entrega, aumenta a diferença com o custo de estoque por entrega; logo, em função da política de abertura de CDUs, novas empresas utilizam os centros, gerando dois efeitos principais: por um lado, a distância média percorrida por cada entrega diminui (Allen et al., 2014) e os custos de transporte também; por outro lado, o custo de estoque aumenta em razão dos custos operacionais dos CDUs e dos custos de estoque. 0 sistema converge para o equilíbrio quando a diferença entre os custos atinge o nível mínimo definido na política (nesse caso, definido arbitrariamente como 10\%).

A variação incremental do número de CDUs utilizados $(d U / d t)$ é determinada pela diferença entre o custo de transporte total $(T)$ e o custo de estoque total (I), obedecendo a relação descrita na Equação 6 , com valor constante de $5 \%$ ao mês. 
A Equação 7, por sua vez, descreve a relação entre a distância média por entrega $\left(L_{d}\right)$ e o número de CDUs $(U)$ e foi determinada por regressão com base em Janjevic et al. (2015) e calibrado com dados de Curitiba, Paraná, Brasil, com a distância máxima na ausência de CDUs sendo de $8 \mathrm{~km}$ para o caso base.

$\frac{d U}{d t}=\left\{\begin{array}{r}0,05, \frac{T}{I} \geq 1,10 \\ 0, \frac{T}{I}<1,10\end{array}\right.$

$L_{d}=8-0,3 . U$

A Figura 8 mostra o comportamento dos custos de transportes, do custo de estoque e do custo logístico calculado pelo modelo, que apresentam padrões consistentes em comparação aos valores teóricos estimados na literatura (Chopra \& Meindl, 2004), conforme a Figura 9.

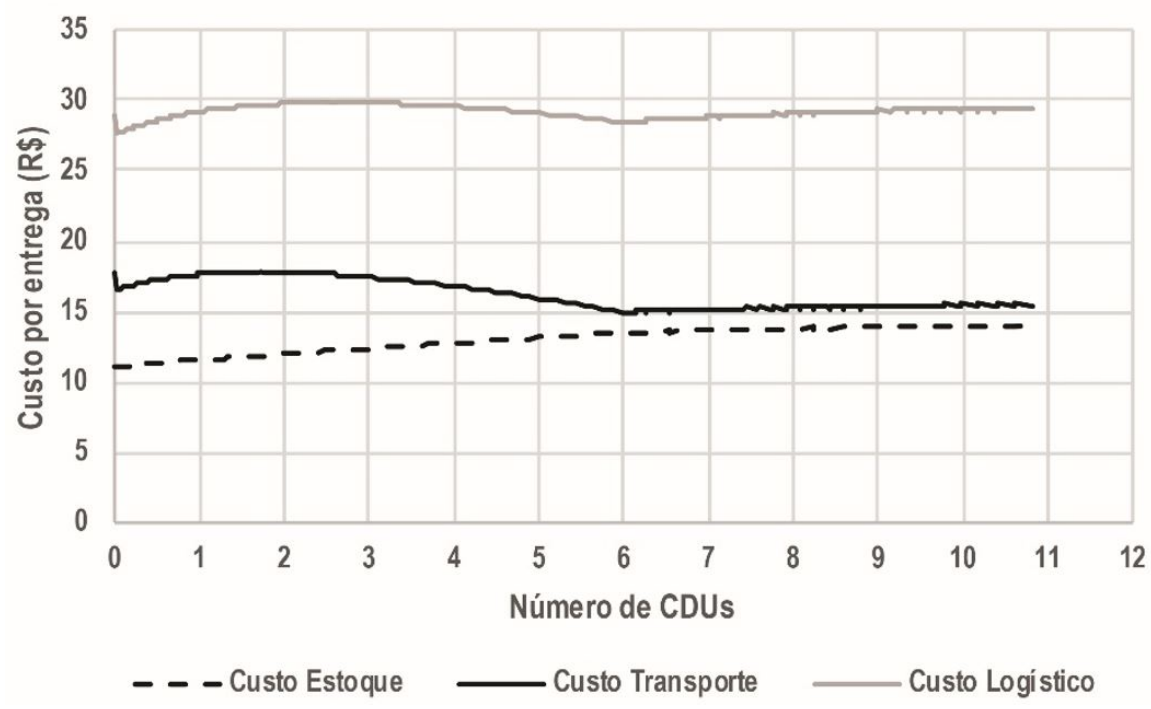

Figura 8 - Custos/número de CDUs (modelo). Fonte: resultados da simulação.

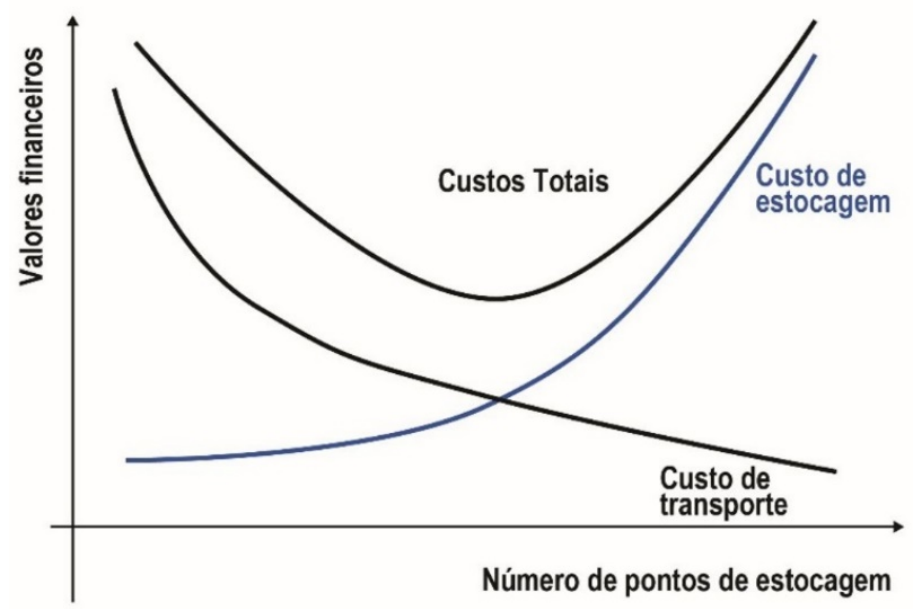

Figura 9 - Custos/número de CDUs (literatura). Fonte: Chopra \& Meindl (2004). 


\section{Premissas e considerações sobre os CDUs}

As premissas adotadas nesse ciclo incluem: i) o abastecimento dos CDUs é feito durante a noite (Panero \& Shin, 2011), o que implica que as viagens para abastecer os CDUs não têm impacto relevante no nível de congestionamento, portanto não são computadas para tal (embora os custos delas foram incluídos com um valor constante); ii) os CDUs estão distribuídos em pontos estratégicos da cidade, otimizando as distâncias aos pontos de entrega; iii) a utilização dos CDUs adiciona em média um dia de estoque ao sistema.

\section{Variáveis de desempenho e políticas avaliadas}

As variáveis de desempenho utilizadas no modelo são: i) as emissões de GEE (medidas em kg equivalentes de $\mathrm{CO}_{2} /$ dia); ii) os custos logísticos totais ( $\mathrm{R} \$ /$ dia), que incluem custo de transporte, armazenagem e estoque. Em seguida, o modelo foi simulado usando quatro configurações de políticas (ver Tabela 2).

Tabela 2 - Políiticas testadas

\begin{tabular}{cccccc}
\hline & Unidade & $\begin{array}{c}\text { Política 1: caso } \\
\text { base }\end{array}$ & $\begin{array}{c}\text { Política 2: } \\
\text { restrições de } \\
\text { caminhões }\end{array}$ & $\begin{array}{c}\text { Política 3: fator } \\
\text { de ocupação }\end{array}$ & $\begin{array}{c}\text { Política 4: } \\
\text { CDUs }\end{array}$ \\
\hline Horas de operação (1) & horas & 10 & 7 & 10 & 10 \\
Fator de ocupação (2) & $\%$ & $70 \%$ & $70 \%$ & $90 \%$ & $80 \%$ \\
Tx. ampliação CDUs (3) & $\% /$ mês & 0 & 0 & 0 & $5 \%$ \\
Distância por entrega (4) & $\mathrm{km}$ & 8 & 8 & 8 & $8-0,3 \mathrm{U}$ \\
Ponto crítico congestionamento (5) & $\%$ & $95 \%$ & $95 \%$ & $95 \%$ & $95 \%$ \\
\hline
\end{tabular}

(1) 10 horas disponíveis para entregas, sendo reduzidas para 7 horas com a política de restrições (das 10h às 17h); (2) Ocupação-padrão de $70 \%$, subindo para $80 \%$ no caso da política de CDUs e para $90 \%$ na política 3, com ações específicas do setor privado; (3) Tomando como referência que o tempo para maturidade operacional de um CDU pode ser de 12 a 18 meses. O modelo considera uma aumento gradual de $5 \%$ ao mês na utilização dos CDUs, com um novo centro aberto ao atingir a capacidade máxima; (4) Para a política de CDUs, as distâncias percorridas por entrega variam segunda a equação $L d=7$ (8-0,3U) (Equação 7), em que U é o número de CDUs (Janjevic et al., 2015). As distâncias são fixas para as outras políticas; (5) medido pela densidade de tráfego, definido pela relação entre o número de veículos/faixa-km e a capacidade das vias. Após atingir o ponto crítico de $95 \%$, são feitos investimentos para ampliar a capacidade, pois o poder público tende a atacar os sintomas do problema de congestionamento e aumentar a capacidade das vias, principalmente em razão das pressões da população (Sterman, 2000).

\section{Cenários avaliados}

Como próximo passo, as quatro políticas e intervenções foram testadas sob dois cenários que representam cidades hipotéticas (Tabela 3). A cidade tipo 1 representa uma cidade com operação logística de pouca intensidade, níveis de congestionamento baixo e custos de operação do CDU relativamente baixo. A cidade tipo 2 representa operação logística intensa, alto nível de congestionamento e custos de operacionais altos. Os dados da cidade 1 foram baseados na cidade de Curitiba, enquanto, para a cidade 2, foram extrapolados com base em Curitiba e complementados com dados da literatura (Browne et al., 2005; Van Duin et al., 2012; CLUB, 2013).

Tabela 3 - Cenários avaliados em cidades hipotéticas

\begin{tabular}{ccccc}
\hline Parâmetro & Unidade & Cidade tipo 1 (3) & Cidade tipo 2 & Cidade tipo 2 \\
\hline \# de pontos de entrega & unidades & 2.000 & 3.000 & $4.000(4)$ \\
\# entregas/dia & entregas & 1.000 & 1.500 & $2.000(4)$ \\
Distância média por entrega (1) & $\mathrm{km}$ & 8 & 12 & $12(5)$ \\
Custo fixo CDU & R $\$$ /mês & 15.000 & 600 & $30.000(6)$ \\
\hline
\end{tabular}


Tabela 3 - Continuação...

\begin{tabular}{ccccc}
\hline Parâmetro & Unidade & Cidade tipo 1 (3) & Cidade tipo 2 & Cidade tipo 2 \\
\hline Nível de congestionamento (2) & $\%$ & $30 \%$ & $40 \%$ & $55 \%(5)$ \\
Velocidade média inicial & $\mathrm{km} / \mathrm{h}$ & 30 & 25 & $15(5)$ \\
\% caminhões & $\%$ & $15 \%$ & $20 \%$ & $30 \%(6)$ \\
\hline
\end{tabular}

(1) valor estabelecido para o cenário base. Esse parâmetro se torna variável nos cenários com CDUs; (2) medido pela densidade de tráfego, definido pela relação entre o números veículos/faixa-km e a capacidade da via; (3) dados coletados em Curitiba, Paraná, Brasil; (4) \# pontos de entrega: Browne et al. (2005); entregas por dia, estimadas de acordo a relação: 1 entrega a cada 2 dias; (5) velocidade média e distância entre entregas: Fortaleza, Brasil de CLUB - Centro de Logística do Brasil (CLUB, 2013); Nível de congestionamento estimado de acordo a função $S=60 \cdot e^{-2 f}$ (Equação 1); (6) custo fixo CDU e percentagem de caminhões foram extrapolados com base em Curitiba.

\section{Resultados das simulações}

Os gráficos das Figuras 10, 11, 12 e 13 apresentam os resultados das simulações para os dois indicadores de desempenho.

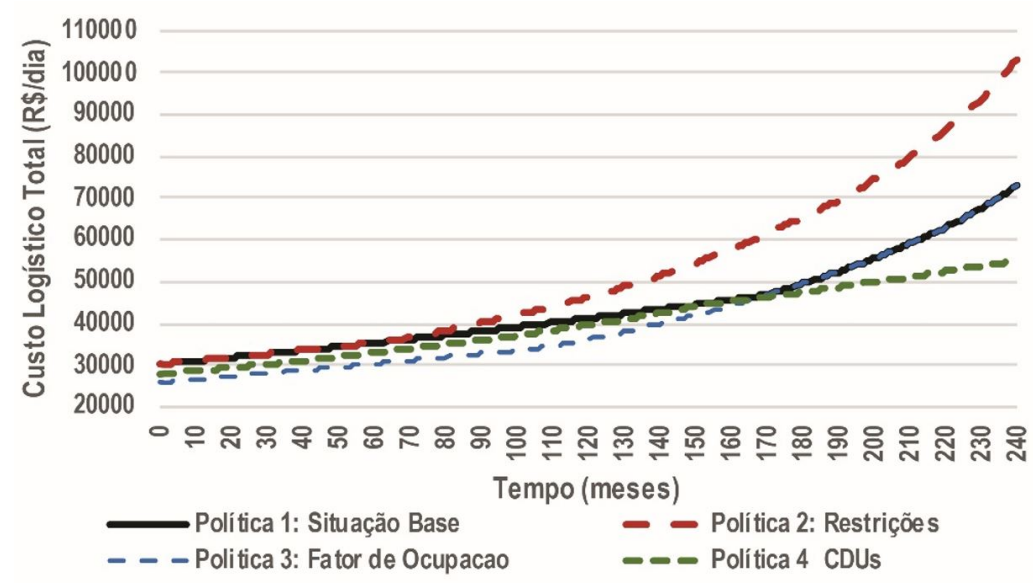

Figura 10 - Custos logísticos cidade tipo 1. Fonte: elaborada pelos autores.

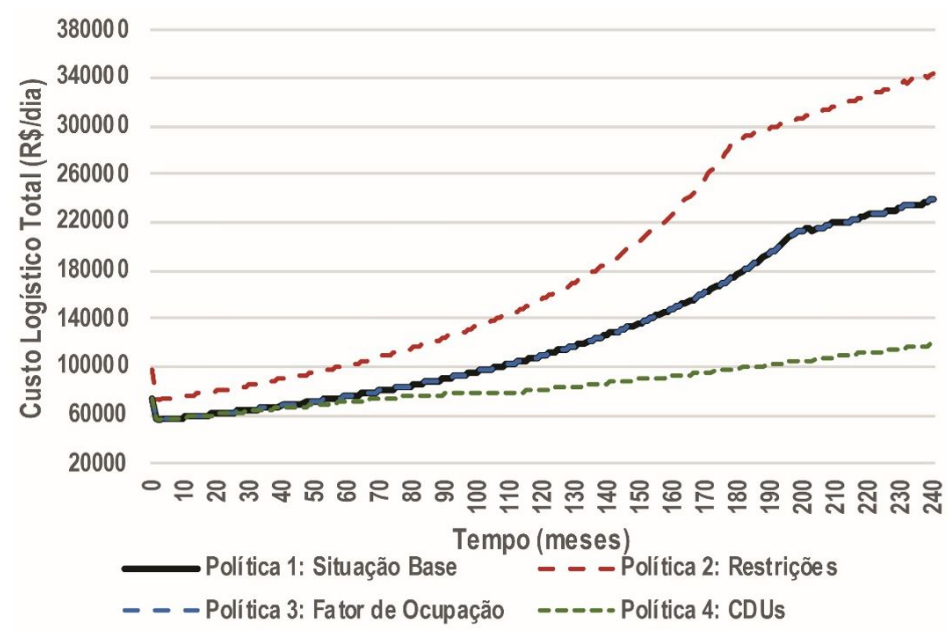

Figura 11 - Custos logísticos cidade tipo 2. Fonte: resultados da simulação. 


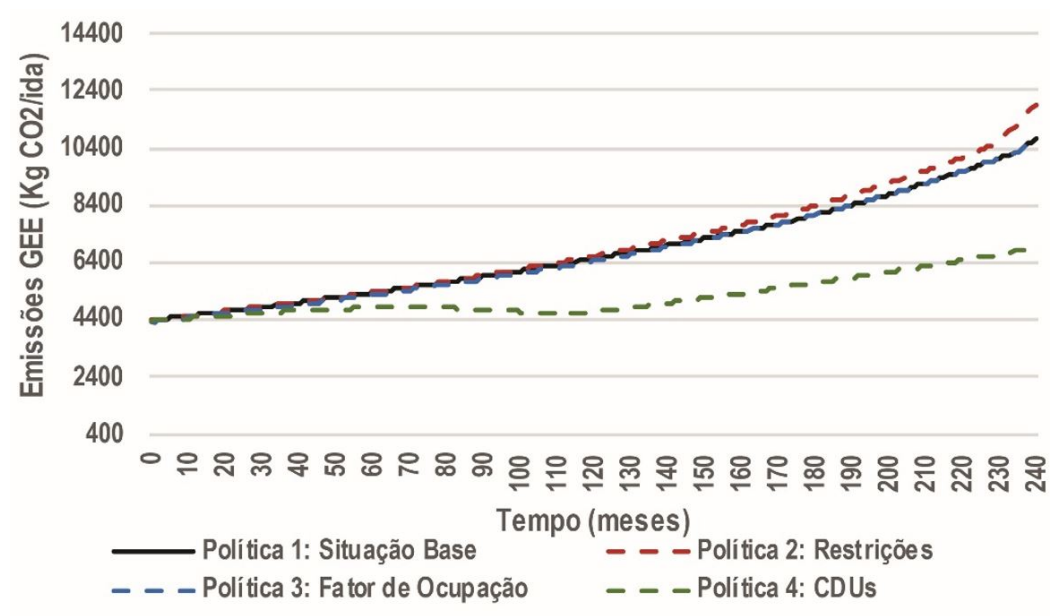

Figura 12 - Emissões de GEE na cidade tipo 1. Fonte: resultados da simulação.

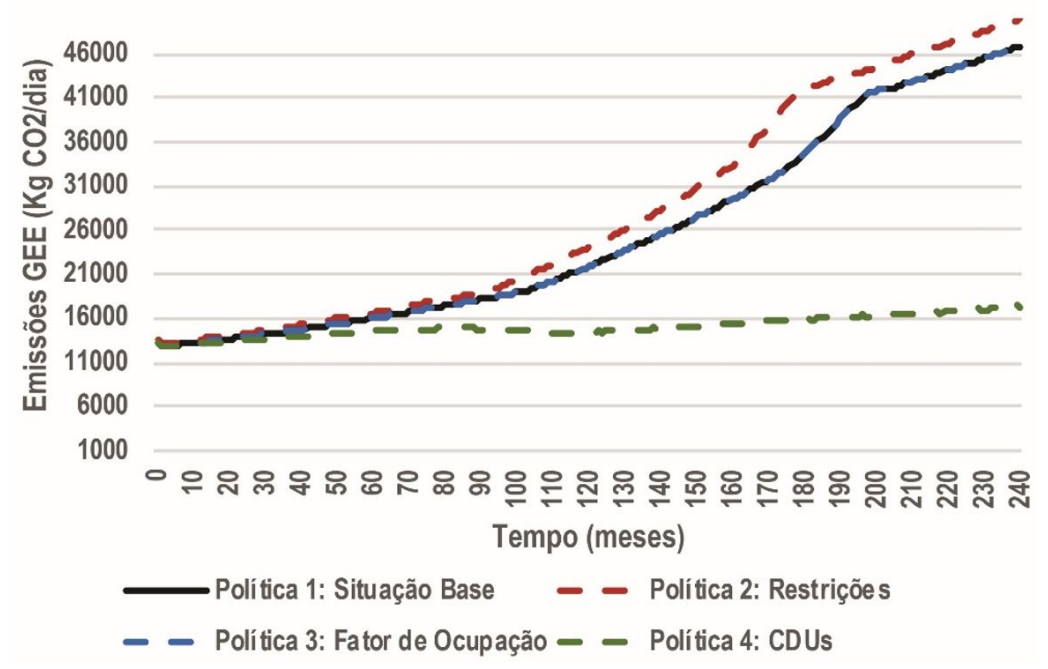

Figura 13 - Emissões de GEE na cidade tipo 2. Fonte: resultados da simulação.

\section{Política 2: impacto negativo das restrições}

A restrição à circulação de veículos de cargas por meio de janelas de tempo (política 2), medida onde os caminhões são proibidos de circular em horários de grande movimento, é adotada em muitas cidades (Allen et al., 2012) e, embora possa reduzir o congestionamento nas horas de pico, aumenta os custos logísticos nos cenários analisados (Figuras 10 e 11). Esse aumento ocorre porque, com a diminuição do tempo disponível para os caminhões realizarem entregas, mais veículos são enviados às ruas para efetuar o mesmo número de entregas. Esse resultado é consistente com outras pesquisas (Yamada \& Taniguchi, 2005; Meyer Sanches et al., 2013). 0 modelo assume que a circulação de caminhões é permitida entre 10h e 17h, horário similar ao adotado na cidade de São Paulo (São Paulo, 2008). 0 fator de ocupação de carga com essa política é reduzido em até $40 \%$ comparativamente ao cenário base. A simulação indica, portanto, um grande impacto das restrições para as empresas privadas, o que explica em parte a resistência que tal política enfrenta (Globo.com, 2016).

Com relação às emissões de GEE, a simulação indica que, para a cidade 1, as restrições geram um leve aumento nos níveis de emissões (Figura 12), os quais, se somados ao benefício da redução dos veículos de carga nos horários críticos (não considerado no modelo), podem, em alguns casos, ter um efeito líquido positivo do ponto de vista ambiental, consistente com alguns estudos (Yamada \& Taniguchi, 2005). Por outro lado, quando essa política é aplicada na cidade tipo 2 , as emissões apresentam um 
aumento de até $20 \%$ considerado com o cenário base (Figura 13), além de que a política de restrições, nesse caso, não traz benefícios econômicos e ambientais. Esse padrão se dá porque, nas cidades onde o sistema de transporte não está saturado, os veículos já estão trabalhando mais próximos de sua capacidade, e a diminuição da janela de entregas impacta menos o número de entregas que cada caminhão pode fazer; em cidades com trânsito intenso, o caminhão está operando em um nível subótimo; nesse caso, a introdução das restrições de horários agrava essa situação.

Nota-se que, nos gráficos da cidade 2, tanto para o cenário base como para o cenário das restrições, há um crescimento exponencial (nos custos logísticos e nas emissões), seguido por um crescimento linear. Esse padrão se dá porque o modelo inclui a premissa de que, ao atingir o nível de $95 \%$ de densidade de tráfego, o que indica uma saturação das vias, o poder público tende aumentar a capacidade das vias, principalmente devido às pressões da população (Sterman, 2000).

\section{Política 3: aumento do fator de ocupação de carga}

A premissa dessa política é que cada empresa busca a otimização das rotas e o aumento do fator de ocupação de carga por meio de um melhor planejamento e do uso de ferramentas de roteirização (Jaller et al., 2015). 0 modelo utiliza uma linha base de $70 \%$ de ocupação, a qual chega a $80 \%$ com a utilização dos CDUs e a $90 \%$ com melhoras no planejamento. Os números adotados são conservadores, já que a literatura destaca casos com linha base de $45 \%$ de ocupação, subindo até $70 \%$ com a utilização dos CDUs (Browne et al., 2005).

De acordo com os resultados da simulação, essa solução permite a redução dos custos logísticos no curto prazo, porém, na cidade tipo 1 (Figura 10), não é robusta, pois, quando o nível de congestionamento atinge níveis críticos, os custos logísticos atingem valores maiores que no caso base. Isso acontece porque, com níveis críticos de congestionamento, não é possível aumentar o número de entregas com um mesmo caminhão, pois não haveria tempo hábil para realizar tais entregas. Um possível cenário no qual a consolidação de carga poderia ser sustentável no longo prazo é quando há colaboração entre empresas (Yang \& Odani, 2006) e um mesmo caminhão realiza entregas de várias delas no mesmo percurso.

\section{Política 4: implantação dos CDUs}

A adoção dos CDUs é a única política que supera o caso base no longo prazo (240 meses). A principal razão é que a introdução dos CDUs muda a estrutura da rede de distribuição, reduzindo as distâncias que os caminhões percorrem para realizar as entregas.

A simulação para a cidade tipo 2 (Figura 11) mostra redução de 8 a $10 \%$ nos custos em relação ao caso base nos primeiros 4 anos, chegando a $35 \%$ no ano 10 e a $60 \%$ de redução no ano 20 . A velocidade média nas vias no ano 20 passa de $8 \mathrm{~km} / \mathrm{h}$ no caso base para $16 \mathrm{~km} / \mathrm{h}$ com os CDUs. Os resultados para a cidade tipo 1, no entanto, não são tão consistentes (Figura 10): nos primeiros 4 anos, são semelhantes à cidade 2 , mas os benefícios continuam abaixo de $10 \%$ até o ano 15 , sendo que o máximo de redução é de $25 \%$. Soma-se a isso o fato que a política 3 - a melhora do fator de ocupação - tem desempenho melhor, nesse caso, até 0 ano 15.

Com relação às emissões, a utilização de CDUs supera todas as outras políticas, mesmo em cidades com níveis de congestionamento baixo (cidade 1) e inclusive no curto prazo (Figura 12); isso se dá porque a redução do custo de transporte é compensada pelo custo adicional de operar os CDUs; no entanto, para as emissões, não há essa contrapartida, então toda a redução contribui para diminuir as emissões. Isso indica que a adoção dos CDUs, se analisada com uma visão integrada que considere também os aspectos ambientais, terá maiores chances de ser implementada. Finalmente, na cidade 2, nota-se que a política de CDUs reduz a necessidade de investimentos em infraestrutura (Figura 13), o que representa um benefício adicional não computado explicitamente no modelo. 


\section{Procedimento proposto para a replicação do modelo}

A seguir, propõe-se um procedimento (Figura 14) para a aplicação do modelo, que contém três passos: coleta de dados de cidade específica; atualização dos parâmetros e corrida do modelo de simulação; análise dos resultados para a cidade específica. Essa primeira etapa do procedimento é chamada de simulação estratégica, pois tem o objetivo de modelar o sistema de logística urbana, analisando o comportamento dos vários subsistemas descritos anteriormente.
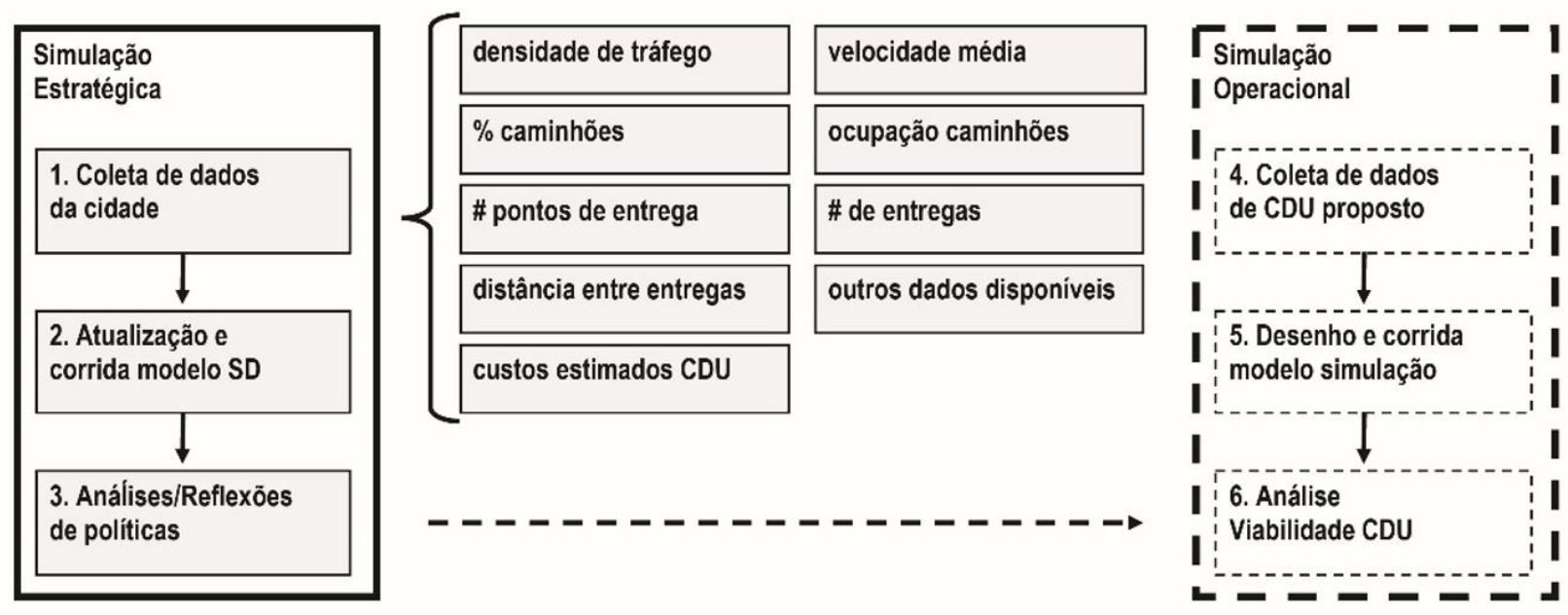

Figura 14 - Procedimento proposto para aplicação do modelo. Fonte: elaborada pelos autores.

Adicionalmente, propõe-se uma segunda etapa no procedimento, que não foi tratada em detalhe neste artigo, mas que se identifica como oportunidade para futuras pesquisas, a qual denomina-se simulação operacional, cujo objetivo é avaliar o modelo de negócio e a viabilidade financeira de um CDU específico e inclui três passos adicionais: coleta de dados de um CDU específico; construção e corrida do modelo de simulação; análise dos resultados e conclusão sobre a viabilidade financeira do CDU.

\section{Conclusões e considerações finais}

\section{Desafios no curto prazo}

O modelo contribuiu para gerar novos insights com relação aos CDUs. Em qualquer dos cenários simulados, os benefícios no curto prazo, de um a dois anos, são difíceis de atingir, pois outras políticas, como o aumento do fator de ocupação de carga - embora precisem ser lideradas pelo setor privado podem gerar resultados mais imediatos, o que ajuda a explicar alguns dos problemas que os gestores públicos têm para justificar os projetos de CDUs.

\section{Visão de longo prazo}

Outra conclusão importante - essa favorável aos CDUs - é que, no longo prazo, os CDUs oferecem uma solução robusta, retardando a saturação da infraestrutura e evitando a necessidade de novos investimentos, e esse comportamento se dá especialmente em cidades que já possuem uma atividade logística mais intensa. A justificativa é que a introdução dos CDUs é a única solução que muda a rede logística, reduzindo as distâncias que os caminhões circulam para realizar as entregas. Outras políticas ou intervenções buscam limitar viagens (política 2) ou otimizar rotas (política 3) e, embora possam 
atingir certo grau de eficiência no curto prazo, em situações extremas são difíceis de manter e podem gerar efeitos colaterais, como o caso das restrições de caminhões, que fazem com que mais veículos sejam necessários. Essa é provavelmente a principal contribuição do presente trabalho.

\section{Características das cidades}

As simulações mostram também que há uma correlação direta entre as características de cada cidade e os benefícios das políticas de logística urbana. Essa conclusão se alinha a casos reais, por exemplo, na cidade de São Paulo, onde os níveis de congestionamento são críticos e onde restrições já foram implementadas, o município lançou um programa-piloto de entregas noturnas (CET, 2015), que pode ser o primeiro passo para viabilizar os CDUs. A cidade de Curitiba, por outro lado, que representa um nível médio de congestionamento e logística menos intensa, implementou medidas de restrições de carga (Globo.com, 2016), mas tem enfrentado barreiras para a implementação de CDUs (IDB, 2013) pela dificuldade de provar seus benefícios.

\section{Localização dos CDUs}

O modelo permite concluir também que a localização dos CDUs é um fator-chave e que a configuração da rede de CDUs deve buscar reduzir as distâncias percorridas pelos veículos, pois essa é a variável mais sensível nos cenários simulados, corroborando a conclusão de outros autores (Crainic et al., 2004; Muñuzuri et al., 2012; Muñoz-Villamizar et al., 2014).

\section{Parcerias público-privadas}

A literatura e as simulações mostram que as políticas de logística urbana não dependem apenas do setor público (como no caso da consolidação de carga), mas, em contraparte, dificilmente são aplicadas apenas pelo setor privado (como no caso dos CDUs), por isso as parcerias público-privadas têm surgido em vários países como solução para a logística urbana (Panero \& Shin, 2011). Nesse contexto, uma vez definido que, no longo prazo, os CDUs podem contribuir para os objetivos buscados, a discussão não deveria centrar-se na sustentabilidade financeira sem dependência do poder público, mas sim em qual o melhor modelo de parceria público-privada para gerenciá-los. Por isso, na segunda etapa do procedimento, propõe-se incluir uma avaliação dos fundamentos operacionais, financeiros e do nível de participação pública e privada que cada CDU deve ter antes de decidir sobre sua implantação.

\section{Modelo e procedimento}

A utilização de dinâmica de sistemas permitiu internalizar variáveis-chave na compreensão mais ampla do sistema e testar vários cenários por um período longo, contrapondo outros modelos que avaliam os resultados de maneira estática ou no curto prazo. A incorporação da política de gestão de estoque, por sua vez, contribuiu para entender os modelos de decisão em relação às frotas, bem como para definir o ritmo de implantação e o número de CDUs para atingir o equilíbrio.

0 modelo proposto e o procedimento para sua implantação são de fácil aplicação e não exigem grande quantidade de dados, os quais podem ser obtidos por meio de pesquisas com base em dados existentes (por exemplo, número de empresas), entrevistas com empresas (por exemplo, número de entregas semanais, tamanho dos lotes, distância entre entregas etc.) ou coleta de dados em campo (por exemplo, velocidade média e densidade de tráfego). Na ausência de dados, podem ser utilizados parâmetros de outras cidades, devidamente ajustados e calibrados mediante análises de sensibilidade. 


\section{Limitações e simplificações do modelo e futuras pesquisas}

O objetivo do modelo é oferecer uma ferramenta estratégica para que os gestores públicos analisem alternativas de políticas em logística urbana, com pouco esforço de coleta de dados, não tendo a pretensão de ser uma ferramenta de previsão, mas sim de apoio para a compreensão do sistema que possa guiar decisões de políticas públicas. Reconhece-se, no entanto, que, conforme os projetos de CDUs avançam, análises mais detalhadas são necessárias e futuras pesquisas podem incluir o desenvolvimento de um modelo de microssimulação para analisar os resultados financeiros do CDU, tomando em conta diferentes esquemas de gestão pública, privada ou público-privada.

Durante o processo de modelagem, algumas simplificações foram feitas: no caso específico das restrições de circulação, o modelo não reflete as diferentes variações a essa política, que podem incluir, por exemplo, flexibilidade de circulação para caminhões menores de 3 toneladas (Meyer Sanches et al, 2013); algumas dessas flexibilizações podem reduzir os impactos ao setor privado e torná-las mais atrativas. Por outro lado, alguns potenciais benefícios dos CDUs não foram incluídos, como a utilização de veículos elétricos (Verlinde et al., 2014), a redução de tempos de carga e descarga e a provisão de serviços de valor agregado (Browne et al., 2005; Allen et al., 2012). Embora possam contribuir para aumentar os benefícios e mudar as decisões de políticas públicas, entende-se que esses benefícios dependem de particularidades de cada caso, por isso optou-se por modelar cenários mais gerais, cujas conclusões possam ser aproveitadas com uma perspectiva mais ampla. Isso, no entanto, não limita que futuras versões do modelo possam incluir essas variações, o que se poderia fazer com relativamente pouco esforço de modelagem.

\section{Referências}

Abbas, K. A., \& Bell, M. G. (1994). System dynamics applicability to transportation modeling. Transportation Research Part A, Policy and Practice, 28(5), 373-390. http://dx.doi.org/10.1016/0965-8564(94)90022-1.

Allen, J., Browne, M., Woodburn, A., \& Leonardi, J. (2012). The role of urban consolidation centres in sustainable freight transport. Transport Reviews, 32(4), 473-490. http://dx.doi.org/10.1080/01441647.2012.688074.

Allen, J., Browne, M., Woodburn, A., \& Leonardi, J. (2014). A review of urban consolidation centres in the supply chain based on a case study approach. Supply Chain Forum, 15(4), 100-112.

Anand, N., Quak, H., Van Duin, R., \& Tavasszy, L. (2012). City logistics modeling efforts: trends and gaps-a review. Procedia: Social and Behavioral Sciences, 39, 101-115. http://dx.doi.org/10.1016/j.sbspro.2012.03.094.

Angerhofer, B. J., \& Angelides, M. C. (2000). System dynamics modelling in supply chain management: research review. In: Winter Simulation Conference Proceedings (pp. 342-351). Orlando: IEEE. http://dx.doi.org/10.1109/WSC.2000.899737.

Browne, M., Allen, S., Andersen, S., Woodburn, A., Taniguchi, E., \& Thompson, R. G. (2005). Urban freight consolidation centres, recent advances in city logistics (pp. 253-265). Amsterdam, Netherlands: Elsevier.

Button, K. J., \& Pearman, A. D. (1981). The economics of urban freight transport. New York: Holmes \& Meier. http://dx.doi.org/10.1007/978-1-349-04151-0.

Centro de Logística Urbana do Brasil - CLUB. (2013). Debates sobre logística urbana brasileira, Banco Mundial. Campinas: CLUB.

Chopra, S., \& Meindl, P. (2004). Supply chain management: strategy: planning and operation. USA: Prentice Hall.

Combes, F. (2014). An empirical evaluation of the EOQ model of choice of shipment size in freight transport. Transportation Research Record: Journal of the Transportation Research Board

Comi, A., Delle Site, P., Filippi, F., \& Nuzzolo, A. (2012). Urban freight transport demand modelling: a state of the art. European Transport, 51, 1-8. 
Companhia de Engenharia de Tráfego - CET. (2015). Programa de Entrega Noturna. Projeto Piloto. Recuperado em 30 de julho de 2017, de http://www.cetsp.com.br/consultas/caminhoes/programa-de-entrega-noturna/projeto-piloto.aspx

Crainic, T. G., Ricciardi, N., \& Storchi, G. (2004). Advanced freight transportation system for congested urban areas. Transportation Research Part C, Emerging Technologies, 12(2), 119-137. http://dx.doi.org/10.1016/j.trc.2004.07.002.

Crainic, T. G., Ricciardi, N., \& Storchi, G. (2009). Models for evaluating planning city logistics systems. Transportation Science, 43(4), 432-454. http://dx.doi.org/10.1287/trsc.1090.0279.

Dablanc, L. (2007). Goods transport in large European cities: difficult to organize, difficult to modernize. Transportation Research Part A, Policy and Practice, 41(3), 280-285. http://dx.doi.org/10.1016/j.tra.2006.05.005.

Fazel, F. (1997). A comparative analysis of inventory costs of JIT and EOQ purchasing. International Journal of Physical Distribution \& Logistics Management, 27(8), 496-504. http://dx.doi.org/10.1108/09600039710182680.

Federal Highway Administration - FHA. (2008). Freight benefit/cost study: phase iii - analysis of regional benefits of highwayfreight improvements. Washington: Office of Freight Management and Operations. Recuperado em 30 de outubro de 2017, de https://ops.fhwa.dot.gov/freight/freight_analysis/econ_methods/freight_cba_study/freight_cba_phase3.pdf

Figliozzi, M. A. (2007). Analysis of the efficiency of urban commercial vehicle tours: data collection, methodology and policy implications. Transportation Research Part B: Methodological, 41(9), 1014-1032. http://dx.doi.org/10.1016/j.trb.2007.04.006.

Friesz, T. L. \& Holguín-Veras, J. (2005). Dynamic game-theoretic models of urban freight: formulation and solution approach. In A. Reggiani \& L. A. Schintler. Methods and models in transport and telecommunications: cross atlantic perspectives (pp. 143-161). Berlin: Springer. http://dx.doi.org/10.1007/3-540-28550-4_8.

Geroliminis, N., \& Daganzo, C. F. (2007). Existence of urban-scale macroscopic fundamental diagrams: some experimental findings. Berkeley: University of California Berkeley Center for Future Urban Transport.

Giuliano, G., \& Dablanc, L. (2013). Approaches to managing freight in metropolitan areas. City Logistics Research: A Transatlantic Perspective, 1, 63-76.

Globo.com. (2016). Limite de peso para tráfego na Linha Verde sobe para dez toneladas. Recuperado em 20 de novembro de 2017, de http://g1.globo.com/pr/parana/transito/noticia/2016/07/limite-de-peso-para-trafego-na-linha-verde-sobepara-dez-toneladas.html

Goodman, R.W. (2005). Whatever you call it, just don't think of last-mile logistics. Global Logistics and Supply Chain Strategies, 1-5.

Holguín-Veras, J., \& Patil, G. R. (2008). A multicommodity integrated freight origin-destination synthesis model. Networks and Spatial Economics, 8(2), 309-326. http://dx.doi.org/10.1007/s11067-007-9053-4.

Holguín-Veras, J., Pérez, N., Cruz, B., \& Polimeni, J. (2006). Effectiveness of financial incentives for off-peak deliveries to restaurants in Manhattan, New York. Transportation Research Record: Journal of the Transportation Research Board, 1966(1), 51-59. http://dx.doi.org/10.1177/0361198106196600107.

Holguín-Veras, J., Silas, M., Polimeni, J., \& Cruz, B. (2008). An investigation on the effectiveness of joint receiver-carrier policies to increase truck traffic in the off-peak hours. Networks and Spatial Economics, 8(4), 327-354. http://dx.doi.org/10.1007/s11067-006-9011-6.

Holguín-Veras, J., Wang, C., Browne, M., Hodge, S. D., \& Wojtowicz, J. (2014). The New York city off-hour delivery project: lessons for city logistics. Procedia: Social and Behavioral Sciences, 125, 36-48.

http://dx.doi.org/10.1016/j.sbspro.2014.01.1454.

Holguín-Veras, J.; Amaya-Leal, J., Wojtowicz, J., Jaller, M., González-Calderón, C., Sánchez-Díaz, I., Wang, X., Haake, D. G., Rhodes, S. S. \& Hodge, S. D. (2015). Improving freight system performance in metropolitan areas: a planning guide (No. 38, NCFRP Report). Washington: Transportation Research Board. http://dx.doi.org/10.17226/22159.

Hunt, J. D., \& Stefan, K. J. (2007). Tour-based microsimulation of urban commercial movements. Transportation Research Part B: Methodological, 41(9), 981-1031. http://dx.doi.org/10.1016/j.trb.2007.04.009.

Instituto de Pesquisa e Planejamento Urbano de Curitiba - IPPUC. (2014). Estudo de Logística Urbana de Curitiba. Curitiba: IPPUC. Recuperado em 2 de março de 2017, de http://www.ippuc.org.br/mostrarpagina.php?pagina=327\&idioma=1\&ampliar=n\%E3o 
Inter-American Development Bank - IDB. (2013). BR-T1165-Support to Improve City Logistics Performance in Curitiba. New York: IDB. Recuperado em 3 de dezembro de 2016, de https://www.iadb.org/en/project/BR-T1165

Jaller, M., Sanchez, S., Green, J., \& Fandiño, M. (2015). Quantifying the impacts of sustainable city logistics measures in the Mexico City Metropolitan Area. In Proceedings ot the 9th International Conference on City Logistics (pp. 613-626). Tenerife, Canary Islands: Elsevier.

Janjevic, M., Lebeau, P., Ndiaye, A. B., Machari, C., Van Mierlo, J., \& Nsamzinshuti, N. (2015). Strategic scenarios for sustainable urban distribution in the Brussels capital. In Proceedings of the 9th International Conference on City Logistics (pp. 598-612). Tenerife, Canary Islands: Elsevier.

Janssen, B. J. P., \& Oldenburger, A. H. (1991). Product channel logistics and city distribution centers: the case of the Netherlands. In Proceedings of the OECD Seminar on Future Road Transport Systems and Infrastructures in Urban Areas (pp. 289-302). Chiba, Japan: OECD.

Karato, K., \& Sato, N. (2009). The speed-density relationship: road traffic flow analysis with spatial panel data, Faculty of Economics. University of Toyama. Graduate School of Public Policy, University of Tokyo.

Kohler, U. (2004). New ideas for the city logistics project in Kassel. In: Logistics Systems for Sustainable Cities: Proceedings of the 3rd International Conference on City Logistics (pp. 321-332). Amsterdam: Elsevier.

Lima, O. F., Jr. (2004). Desempenho em serviços de transportes: conceitos, métodos e práticas. Campinas: Faculdade de Engenharia Civil, Universida de de Campinas.

Lindholm, M. (2013). Urban freight transport from a local authority perspective: a literature review. Trasporti Europei, 54(3), 1-37.

Marcucci, E., \& Danielis, R. (2008). The potential demand for an urban freight consolidation centre. Transportation, 35(2), 269-284. http://dx.doi.org/10.1007/s11116-007-9147-3.

Meyer Sanches, L., Pinto, J. A., \& Fontes, L. J. (2013). Freight vehicle circulation restriction policy in an emerging country metropolitan area: undesired impacts. In Proceedings of the 31st International Conference of the System Dynamics Society (pp. 3370-3382). Cambridge, Massachusetts: Curran Associates.

Muñoz-Villamizar, A. F., Montoya-Torres, J. R., \& Herazo-Padilla, N. (2014). Mathematical programming modeling and resolution of the location-routing problem in urban logistics. Ingeniería y Universidad, 18(2), 271-289.

http://dx.doi.org/10.11144/Javeriana.IYU18-2.mpmr.

Muñuzuri, J., Cortés, P., Grosso, R., \& Guadix, J. (2012). Selecting the location of minihubs for freight delivery in congested downtown areas. Journal of Computational Science, 3(4), 228-237. http://dx.doi.org/10.1016/j.jocs.2011.12.002.

Muñuzuri, J., Cortés, P., Onieva, L., \& Guadix, J. (2010). Modeling peak-hour urban freight movements with limited data availability. Computers \& Industrial Engineering, 59(1), 34-44. http://dx.doi.org/10.1016/j.cie.2010.02.013.

Ogden, K. W. (1992). Urban goods movement: a guide to policy and planning. Aldershot: Ashgate.

Oliveira, L. K., Nunes, N. T. R., \& Novaes, A. G. N. (2010). Assessing model for adoption of new logistical services: an application for small orders of goods distribution in Brazil. Procedia: Social and Behavioral Sciences, 2(3), 6286-6296. http://dx.doi.org/10.1016/j.sbspro.2010.04.038.

Oppenheim, N. (1993). A combined, equilibrium model of urban personal travel and goods movements. Transportation Science, 27(2), 161-171. http://dx.doi.org/10.1287/trsc.27.2.161.

Panero, M., \& Shin, H.-S. (2011). Urban distribution centers, a means to reducing freight vehicle miles traveled. New York: Nyu Rudin Center for Transportation Policy and Management.

Quak, H., \& De Koster, R. (2006). The impacts of time access restrictions and vehicle weight restrictions on food retailers and the environment. European Journal of Transport and Infrastructure Research, 6(2), 131-150.

Quak, H., \& Tavasszy, L. (2011). Customized solutions for sustainable city logistics: the viability of urban freight consolidation centres. In J. van Nunen \& P. Huijbregts \& P. Rietveld. Transitions towards sustainable mobility (pp. 213-233). Springer. http://dx.doi.org/10.1007/978-3-642-21192-8_12. 
Qureshi, A. G., Taniguchi, E., \& Yamada, T. (2013). Evaluation of truck ban schemes using exact optimization for vehicle routing problem with time windows. In Proceedings of the Transportation Research Board - 92nd Annual Meeting. Washington: The National Academy Press. Recuperado em 3 de dezembro de 2016, de https://trid.trb.org/Results?q=\&serial=\%22Transportation\%20Research\%20Board\%2092nd\%20Annual\%20Meeting\%22

Rodrigue, J., Comtois, C., \& Slack, B. (2013). The geography of transport systems. Routledge: Taylor \& Francis Group. http://dx.doi.org/10.4324/9780203371183.

Ruske, W. (1994). City logistics solutions for urban commercial transport by cooperative operations management. In: Proceedings of the OECD Seminar on Advanced Road Transport Technologies (pp. 195-228). Omiya, Japan: OECD.

São Paulo. (2008, 24 de julho). Decreto n. 49.800, de 23 de Julho de 2008. Regulamenta a Lei no 14.751, de 28 de maio de 2008 , que dispõe sobre a implantação do Programa de Restrição ao Trânsito de Veículos Automotores Pesados, do tipo caminhão, no Município de São Paulo. São Paulo: Diário Oficial da Cidade de São Paulo.

Shepherd, S. P. (2014). A review of system dynamics models applied in transportation. Transportmetrica B: Transport Dynamics, 2(2), 83-105.

Sterman, J. D. (2000). Business dynamics: system thinking and modeling for a complex world. Boston: Irwin McGraw-Hill.

Taniguchi, E., \& Thompson, R. G. (2002). Modeling city logistics. Transportation Research Record: Journal of the Transportation Research Board, 1790, 45-51. http://dx.doi.org/10.3141/1790-06.

Taniguchi, E., Noritake, T., Yamada, T., \& Izumitani, T. (1999). Optimal size and location planning of public logistics terminals. Transportation Research, 35(3), 207-222. http://dx.doi.org/10.1016/S1366-5545(99)00009-5.

Taniguchi, E., Thompson, R. G., \& Yamada, T. (2012). Emerging techniques for enhancing the practical application of city logistics models. Procedia: Social and Behavioral Sciences, 39, 3-18. http://dx.doi.org/10.1016/j.sbspro.2012.03.087.

Taniguchi, E., Thompson, R. G., \& Yamada, T. (2014). Recent trends and innovations in modelling city logistics. In Proceedings of the 8th International Conference on City Logistics (Vol. 125, pp. 4-14). Bali: Elsevier.

Thaller, C., Niemann, F., Dahmen, B., Clausen, U., \& Leerkamp, B. (2016). Describing and explaining urban freight transport by System Dynamics. World Conference on Transport Research, Shanghai. Transportation Research Procedia, 25, $1075-1094$. http://dx.doi.org/10.1016/j.trpro.2017.05.480.

Thompson, R. G. (2015). Vehicle orientated initiatives for improving the environmental performance of urban freight systems. In B. Fahimnia, M. G. H. Bell, D. A. Hensher, J. Sarkis. Green logistics and transportation (pp. 119-129). USA: Springer.

Van Duin, J. H. R. (1997). Evaluation and evolution of the city distribution concept. In L. J. Sucharov \& G. Bidini. Transport and the environment for the 21st century III (pp. 327-337). Southampton, UK: WIT Press.

Van Duin, J. H. R., Quak, H., \& Muñuzuri, J. (2010). New challenges for urban consolidation centres: a case study in Hague. Procedia: Social and Behavioral Sciences, 2(3), 6177-6188. http://dx.doi.org/10.1016/j.sbspro.2010.04.029.

Van Duin, J. H. R., Van Kolck, A., Anand, N., Tavasszy, L. A., \& Taniguchi, E. (2012). Towards agent-based modelling approach for the evaluation of dynamic usage of urban distribution centres. Procedia: Social and Behavioral Sciences, 39, 333-348. http://dx.doi.org/10.1016/j.sbspro.2012.03.112.

Verlinde, S., Macharis, C., Milan, L., \& Kin, B. (2014). Does a mobile depot make urban deliveries faster, more sustainable and more economically viable? results of a pilot test in Brussels. Transportation Research Procedia, 4, 361-373.

http://dx.doi.org/10.1016/j.trpro.2014.11.027.

Visser, J., Nemoto, T., \& Browne, M. (2014). Home delivery and the impacts on urban freight transport: a review. Procedia: Social and Behavioral Sciences, 125, 15-27. http://dx.doi.org/10.1016/j.sbspro.2014.01.1452.

Visser, J., van Binsbergen, A., \& Toshinori, N. (1999). Urban freight transport policy and planning. In Proceedings of the 1st International Symposium on City Logistics (pp. 58-71). Cairns, Australia: Institute of City Logistics.

Weltevreden, J. W. (2008). B2C e-commerce logistics: the rise of collection-and-delivery points in the Netherlands. International Journal of Retail \& Distribution Management, 36(8), 638-660. http://dx.doi.org/10.1108/09590550810883487. 
Wisetjindawat, W., Sano, K., Matsumoto, S., \& Raothanachonkun, P. (2007). Microsimulation model for modelling freight agents interactions in urban freight movement. In Proceedings of the TRB 2007 Annual Meeting. Washington: National Academies of Sciences, Engineering, and Medicine. Recuperado em 3 de dezembro de 2016, de https://trid.trb.org/Results?q=\&serial="Transportation\%20Research\%20Board\%2086th\%20Annual\%20Meeting"

Wolpert, S., \& Reuter, C. (2012). Status quo of city logistics in scientific literature: a systematic literature review. Transportation Research Record: Journal of the Transportation Research Board, 2269(1), 110-116.

http://dx.doi.org/10.3141/2269-13.

Yamada, T., \& Taniguchi, E. (2005). Modelling the effects of city logistics schemes. In E. Taniguchi \& R. G. Thompson (Eds.), Recent Advances in City Logistics - Proceedings of the 4th International Conference on City Logistics (pp. 75-89). Kidlington, Oxford: Elsevier Ltd.

Yang, D., \& Odani, M. (2006). Analysis on movement of profit for the partner companies in joint delivery system. Proceedings of Infrastructure Planning, 33, 327.

Editor: Fábio Duarte

Recebido: Set. 062017

Aprovado: Ago. 062018 
Anexo 1 - Modelo completo de simulação (Software Vensim PLE x32 versão 7.1)
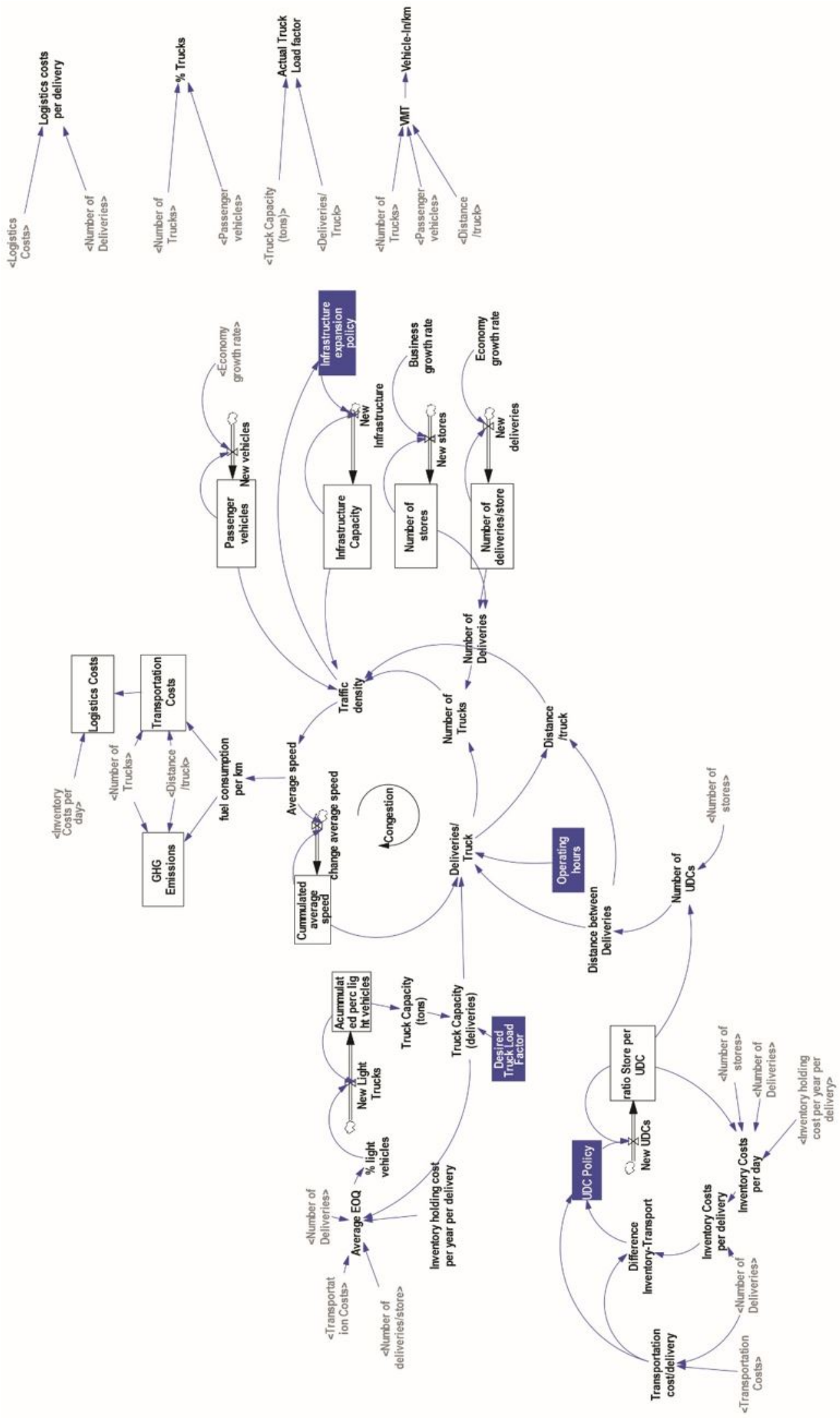